\title{
Survival in second degree atrioventricular block
}

Sir,

The important paper by Shaw et al (1985;53: 587-93) showed that elderly patients with persistent type I second degree heart block have a poor prognosis. They concluded that in the absence of special circumstances these patients should receive permanent pacemakers.

Indications for pacemaker implantation have changed and we need to consider the findings of Shaw $e t$ al in the light of current practice. Thirty of their 77 patients with type I block survived to develop complete heart block and in many centres would have received pacemakers. Thirty five patients had experienced Adams-Stokes attacks during follow up; however, only 33 patients received pacemakers, in 29 cases for disturbance of consciousness or other major cardiac symptoms. Thus as many as 13 of 77 patients remained without pacemakers despite having symptoms of disturbed consciousness that may have been bradycardia related. Furthermore, at entry $25 \%$ of patients had cardiac dyspnoea and $23 \%$ had angina, both symptoms that may have reflected inadequate chronotropic responses to exercise and might be amenable to modern pacemaker treatment.

Before advising permanent pacemaker implantation in these patients we need to be reassured that their sudden deaths were more likely to have been caused by progression of conduction disorder than from other causes. Serious concurrent medical conditions in their group I patients included previous myocardial infarction $(16 \%)$, rheumatic fever $(12 \%)$, diabetes mellitus $(6 \%)$, stroke $(12 \%)$, and rheumatoid arthritis $(13 \%)$. Was the favourable outcome in paced group I patients the result of pacing or freedom from other medical conditions?

Shaw and colleagues have demonstrated that in some patients type I heart block is a predictor of progressive conduction disorder. These patients require careful evaluation and follow up, which will certainly involve ambulatory monitoring and possibly evaluation of chronotropic responses to exercise. This valuable study has led us to change our views on the management of these patients but has not yet convinced us that they should all receive pacemakers at presentation.

R D S Watson,

P N Patel,

Department of Cardiology,

Dudley Road Hospital,

Birmingham B18 7QH.
This letter was shown to the authors, who reply as follows:

Sir,

We thank Dr Watson and Dr Patel for their interest in our paper on chronic second degree atrioventricular block. In answer to their question about the distribution of serious concurrent medical conditions in patients with type I block-for those paced the figures were myocardial infarction four $(12 \%)$, rheumatic fever four $(12 \%)$, diabetes two $(6 \%)$, cerebral vascular accident five $(15 \%)$, and arthritis five $(15 \%)$. For the non-paced patients the figures were myocardial infarction eight $(18 \%)$, rheumatic fever five $(11 \%)$, diabetes three $(7 \%)$, cerebral vascular accident four $(9 \%)$, and arthritis five $(11 \%)$. The only appreciable difference in the frequency of risk factors in favour of the paced group is for myocardial infarction, but even here the numbers are very small, with the difference amounting to only four patients.

We concluded that our data implied that patients with chronic type I second degree block had a similar survival to that of those with type II block (and by inference to third degree block). Watson and Patel comment that patients with third degree block "in many centres would have received pacemakers", and the Bethesda task force suggest that pacing would be appropriate both in third degree and type II second degree block. ${ }^{1}$ Not all patients with these two forms of block do badly; indeed several who for one reason or another were not paced have survived for ten years or more. The difficulty is to spot this minority of potential survivors early on. In the mid and late 1970s we hoped that the results of His bundle electrocardiography would help in the selection of patients for pacing, but having carried out the procedure in 320 patients with a range of conduction disorders we concluded that it was not very useful. We do not quarrel with the recommendation that patients with type I second degree block should be evaluated fully; indeed this is one group in which we would consider going back to recording His bundle electrograms perhaps with a particular search for the presence of split His potentials. ${ }^{2}$ We also agree with the suggestion that pre-pacing exercise testing would be helpful, and this has become part of our standard protocol in recent years.

Some forms of type I block, such as those associated with digitalis intoxication and the high vagal drive of athletic training, are likely to have a good 